\title{
Ein Satz von De Morgan-Sylvester über die Zerlegung der ganzen Zahlen.
}

Von Joh. Zuchristian in Innsbrtuck.

Herr J. J. Sylvester hat in seiner Abhandlung: "On Subinvariants i. e. Semi-Invariants to Binary Quantics of an Unlimited Order" (American Journal of Mathematics V. 5., p. $79 \mathrm{ff}$.) auf Grund der Cayley'schen Theorie der Zerlegung der ganzen Zahlen gezeigt, dass die Anzahl der Darstellungen einer ganzen Zahl $n$ als Summe von drei beliebigen (gleichen oder verschiedenen) ganzen Zahlen gleich der an $\frac{n^{2}}{12}$ zunächstliegenden ganzen Zahl $\left\{\frac{n^{2}}{12}\right\}$ ist.

Herr Prof. Dr. L. Gegenbau er hat von den Mitgliedern der von ihm geleiteten Abtheilung des mathematischen Seminars an der k. k. Universität in Innsbruck einen mit möglichst einfachen Mitteln auszuführenden Beweis dieses interessanten Theorems und die Ermittelung neuer Sätze derselben Kategorie verlangt. In den folgenden Zeilen werde ich nun einen äuferst elementaren Beweis des obigen Satzes angeben und zugleich für die Anzahl der Darstellungen durch vier Elemente einfache Formeln ableiten.

\section{§. 1 .}

Nach einem bekannten Satze von Euler ist die Anzahl der Zerlegungen von $n$ in $x$ beliebige ganze Zahlen gleich der Anzahl $(n-x)$. der Darstellungen von $(n-x)$ durch die Zahlen $1,2, \ldots x$, und es besteht die Relation

$$
\begin{aligned}
n_{x}=n_{x-1}+(n-x)_{x-1} & +\ldots+(n-\alpha x)_{x-1} \\
& \left(x>n-\alpha x>0 ;(o)_{x}=1\right) .
\end{aligned}
$$


Der Gleichung

$$
n=x_{1}+2 x_{2}
$$

genügen folgende ganzzahlige, nicht negative Wertepaare

$$
\begin{aligned}
& x_{1}=n, n-2, n-4, \ldots, n-2 \alpha \\
& x_{2}=0, \quad 1, \quad 2, \ldots, \quad \alpha \\
& \quad(2>n-2 \alpha \geq 0),
\end{aligned}
$$

und demnach ist

$$
n_{2}=\alpha+1
$$

oder, da a gleich der in $\frac{n}{2}$ enthaltenen gröften ganzen Zahl $\left[\frac{n}{2}\right]$ ist

$$
n_{2}=\left[\frac{n}{2}\right]+1
$$

Aus (1) und (2) folgt

$$
\begin{aligned}
(n+6)_{3}-n_{3} & =\left[\frac{n}{2}\right]+\left[\frac{n+1}{2}\right]+6 \\
& =n+6 .
\end{aligned}
$$

Da nun, wie man sofort sieht,

$$
\left\{\frac{(n+9)^{2}}{12}\right\}-\left\{\frac{(n+3)^{2}}{12}\right\}=n+6
$$

ist, so besteht die Beziehung

$$
(n+6)_{3}-n_{3}=\left\{\frac{(n+9)^{2}}{12}\right\}-\left\{\frac{(n+3)^{2}}{12}\right\} .
$$

Setzen wir in derselben für $n$ der Reihe nach

$$
n, n-6, n-2.6, \ldots, n-\left[\frac{n}{6}\right] .6
$$

und addieren die so erhaltenen Gleichungen, so ergibt sich die Gleichung:

$$
(n+6)_{3}-\lambda_{3}=\left\{\frac{(n+9)^{2}}{12}\right\}-\left\{\frac{(\lambda+3)^{2}}{12}\right\}
$$

wo $\lambda$ der Rest ist, welcher bei der Division von $n$ durch 6 verbleibt. Beachten wir, dass 


\begin{tabular}{|c|c|c|}
\hline$\lambda$ & $\lambda_{3}$ & $\left\{\frac{(\lambda+3)^{2}}{12}\right\}$ \\
\hline 0 & 1 & 1 \\
1 & 1 & 1 \\
2 & 2 & 2 \\
3 & 3 & 3 \\
4 & 4 & 4 \\
5 & 5 & 5 \\
\hline
\end{tabular}

also

$$
\lambda_{3}=\left\{\frac{(\lambda+3)^{2}}{12}\right\}
$$

ist, so erhalten wir aus (3)

$$
n_{3}=\left\{\frac{(n+3)^{2}}{12}\right\}
$$

welche Gleichung dem im Anfange erwähnten Satz von De MorganSylvester ausspricht.

\section{\$. 2.}

Nach (1) isst

$$
n_{4}-(n-12)_{4}=n_{3}+(n-4)_{3}+(n-8)_{3}
$$

und daher nach dem Satze von De Morgan-Sylvester für $n=12 s+x-4$

$$
\begin{array}{r}
n_{4}-(n-12)_{4}=\left\{\frac{(12 s+x-1)^{2}}{12}\right\}+\left\{\frac{(12 s+x-5)^{2}}{12}\right\}+\left\{\frac{(12 s+x-9)^{2}}{12}\right\} \\
=36 s^{2}+6(x-5) s+\left\{\frac{(x-1)^{2}}{12}\right\}+\left\{\frac{(x-5)^{2}}{12}\right\}+\left\{\frac{(x-9)^{2}}{12}\right\} \\
(n \geq 12) .
\end{array}
$$

Nun ist für jede Zahl $\mu$, welche nach dem Modul 2 congruent $x$ ist,

$$
\begin{gathered}
{\left[\frac{(n+\mu+4)^{3}}{144}\right]-\left[\frac{(n+\mu-8)^{3}}{144}\right]=} \\
=36 s^{2}+6(x+\mu-6) s+\left[\frac{(x+\mu)^{3}}{144}\right]+\left[\frac{(x+\mu-12)^{3}}{144}\right]
\end{gathered}
$$

somit 


$$
\begin{gathered}
n_{4}-(n-12)_{4}= \\
=\left[\frac{(n+\mu-4)^{3}}{144}\right]-\left[\frac{(n+\mu-8)^{3}}{14 t}\right]-6(\mu-1) s+r
\end{gathered}
$$

wo

$r=\left\{\frac{(x-1)^{2}}{12}\right\}+\left\{\frac{(x-5)^{2}}{12}\right\}+\left\{\frac{(x-9)^{2}}{12}\right\}-\left[\frac{(x+\mu)^{3}}{144}\right]+\left[\frac{(x+\mu-12)^{3}}{144}\right]$

ist.

$$
(n \geq 12)
$$

Setzt man $\mu=1$, bezw. 2, je nachdem $*$ ungerade oder gerade ist, so ergeben sich für $r$ der Reihe nach die Werte:

$$
2,-1,1,-1,0,-1,-1,-1,-2,-1,-3
$$

sodass also nimmt:

a) für ungerade * die letzte Gleichung folgende Gestalt an-

$$
n_{4}-(n-12)_{4}=\left[\frac{(n+5)^{3}}{144}\right]-\left[\frac{(n-7)^{3}}{144}\right]-1
$$

Setzen wir in dieser Formel für $n$ der Reihe nach

$$
n, n-12, \ldots n-12(s-2),
$$

und addieren die dadurch entstehenden Gleichungen, so gelangen wir zur Relation

$$
n_{4}-(x+8)_{4}=\left[\frac{(n+5)^{3}}{144}\right]-\left[\frac{(x+13)^{3}}{144}\right]-\left[\frac{n-8}{12}\right] .
$$

Nun ist aber, wie man sofort sieht, für ungerade $x$

$$
\begin{aligned}
(x+8)_{4}=\left[\frac{(x+13)^{3}}{144}\right]-\varepsilon & \\
& \left(\begin{array}{ll}
\varepsilon=1 & x<11 \\
\varepsilon=2 & x=11
\end{array}\right)
\end{aligned}
$$

und daher

$$
n_{4}=\left[\frac{(n+5)^{3}}{144}\right]-\left[\frac{n+5}{12}\right] .
$$

Nachdem diese Formel auch für

$$
n=1,3,5,7,9,11
$$

besteht, so gilt sie allgemein. 
b) Für gerade Werte von $x$ ergeben sich auf demselben Wege die Beziehungen

wo

$$
\begin{aligned}
& n_{4}-(n-12)_{4}=\left[\frac{(n+6)^{3}}{144}\right]-\left[\frac{(n-6)^{3}}{144}\right]-6 s+r \\
& n_{4}-(x+8)_{4}=\left[\frac{(n+6)^{3}}{144}\right]-\left[\frac{(x+14)^{3}}{144}\right]+m,
\end{aligned}
$$

$$
m=-3 s^{2}-\frac{x+2}{2} s+\frac{x+8}{2}
$$

ist. Nun ist aber für gerade $x$

$$
\begin{gathered}
(x+8)_{4}=\left[\frac{(x+14)^{3}}{144}\right]+\frac{x+8}{2}+\left[\frac{(x+2)^{2}}{48}\right]-\eta \\
\left(\begin{array}{l}
\eta=0 \quad x \geq 8 \\
\eta=1 \quad x=8
\end{array}\right) \\
3 s^{2}+\frac{x+2}{2} s+\left[\frac{(x+2)^{2}}{48}\right]=\left[\frac{(n+6)^{2}}{48}\right]
\end{gathered}
$$

und daher schlieflich

$$
n_{4}=\left[\frac{(n+6)^{3}}{144}\right]-\left[\frac{(n+6)^{2}}{48}\right]+\eta .
$$

Da diese Relation auch für

$$
n=0,2,4,6,8,10
$$

besteht, so besteht sie allgemein.

Wir gelangen somit zu folgendem Satze:

Die Anzahl der Zerlegungen einer ganzen Zahl $u$ in vier beliebige ganze Zahlen ist

oder

$$
\left[\frac{(n+1)^{3}}{144}\right]-\left[\frac{n+1}{12}\right]
$$

$$
\begin{array}{r}
{\left[\frac{(n+2)^{3}}{144}\right]-\left[\frac{(n+2)^{2}}{48}\right]+\eta} \\
\left(\begin{array}{l}
\eta=0 \quad x \gtrless 8 \\
i=1 \quad x=8
\end{array}\right)
\end{array}
$$

je nachdem $n$ nach dem Modul 12 einer ungeraden oder geraden Zahl $x$ congruent ist. 